\title{
Ultrasound guided paravertebral catheter for postoperative multimodal analgesia in thymectomy surgery by VATS approach
}

\author{
Melo MC, Parera A, Trujfllo JC, Martínez E, Unzueta M ${ }^{\mathrm{a}}$ C, Català E. \\ Hospital de la Santa Creu i Sant Pau. Barcelona
}

\section{Background ans Goal of Study}

Thymectomy is performed in myasthenia gravis patients to relief symptoms or reduce medication. 1,2 Video-assisted thoracoscopic (VATS) approach was recently introduced for thymectomy at our center instead of sternotomy. The first patients were treated by conventional postoperative analgesia, but in our VATS experience the postoperative pain is important.4 The objective of our study is to identify if this technique really required multimodal analgesia like other VATS surgeries.

\section{Materials and Method}

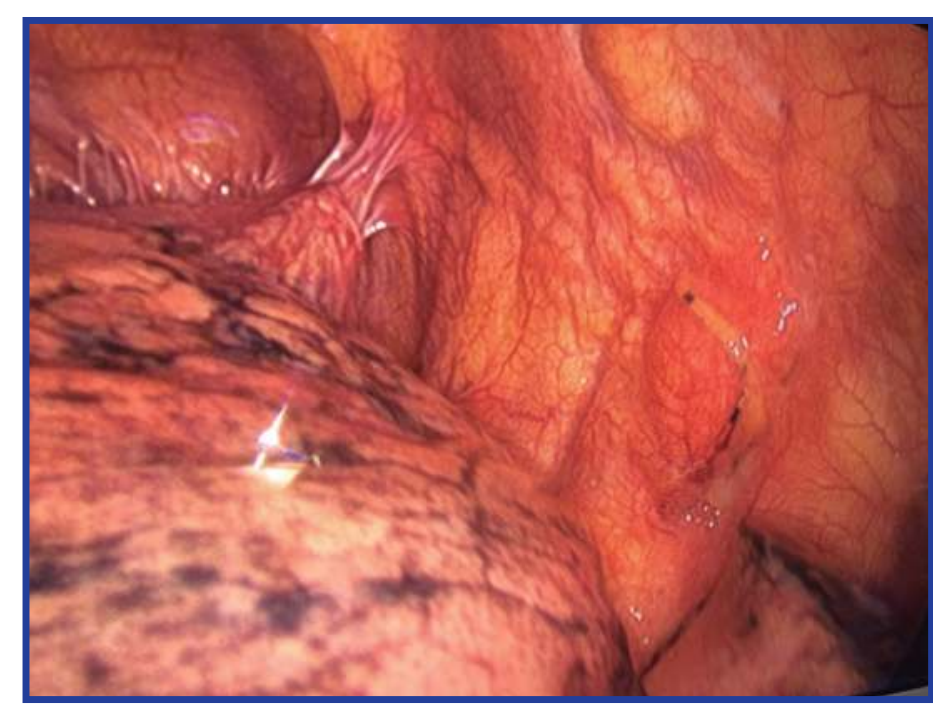

We analyzed retrospectively the series of the first cases performed during 2017. A review of analgesia quality was recorded using Verbal Numerical Rating Scale (VNRS) registered at the end of the surgery, first and second day after surgery and the average of opioid rescue needed in the first 24 hours. Multimodal regimen analgesia was given using our center protocol, using ultrasound guided catheter for continuous paravertebral block (CPBV) - infusion with Ropivacaine 0,375\%, non-steroidal analgesics (NSAIDs) and opioid analgesia as needed.

\section{Result and Discussion}

12 patients were included (42\% Male, $58 \%$ Female), 5 of them were done under general anesthesia (group $\mathrm{A}$ ) and the rest combined general anesthesia and CPBV as multimodal analgesia (group B).

The VPNS media was 6,5 (SD 2,2) at the end of the surgery 3,0 (SD 2,4) at the first day and 2,1 $(1,4)$ at the second day in group $A$. The VPNS media was 5,6 (SD 2,7) at the end of the surgery, 3,2 (SD $1,8)$ at the first day and $1,8($ SD 1,8$)$ at the second day in group $B$.

Only 4 patients (57\%) of the 7 patients in group $B$ needed rescue analgesia in contrast to $4(80 \%)$ of the 5 patients in group A. No complications related to the analgesic technique were found in any groups.

Regarding the pain assessed at three months after surgery, there were no differences between the two groups, they were asymptomatic for pain at surgical site.
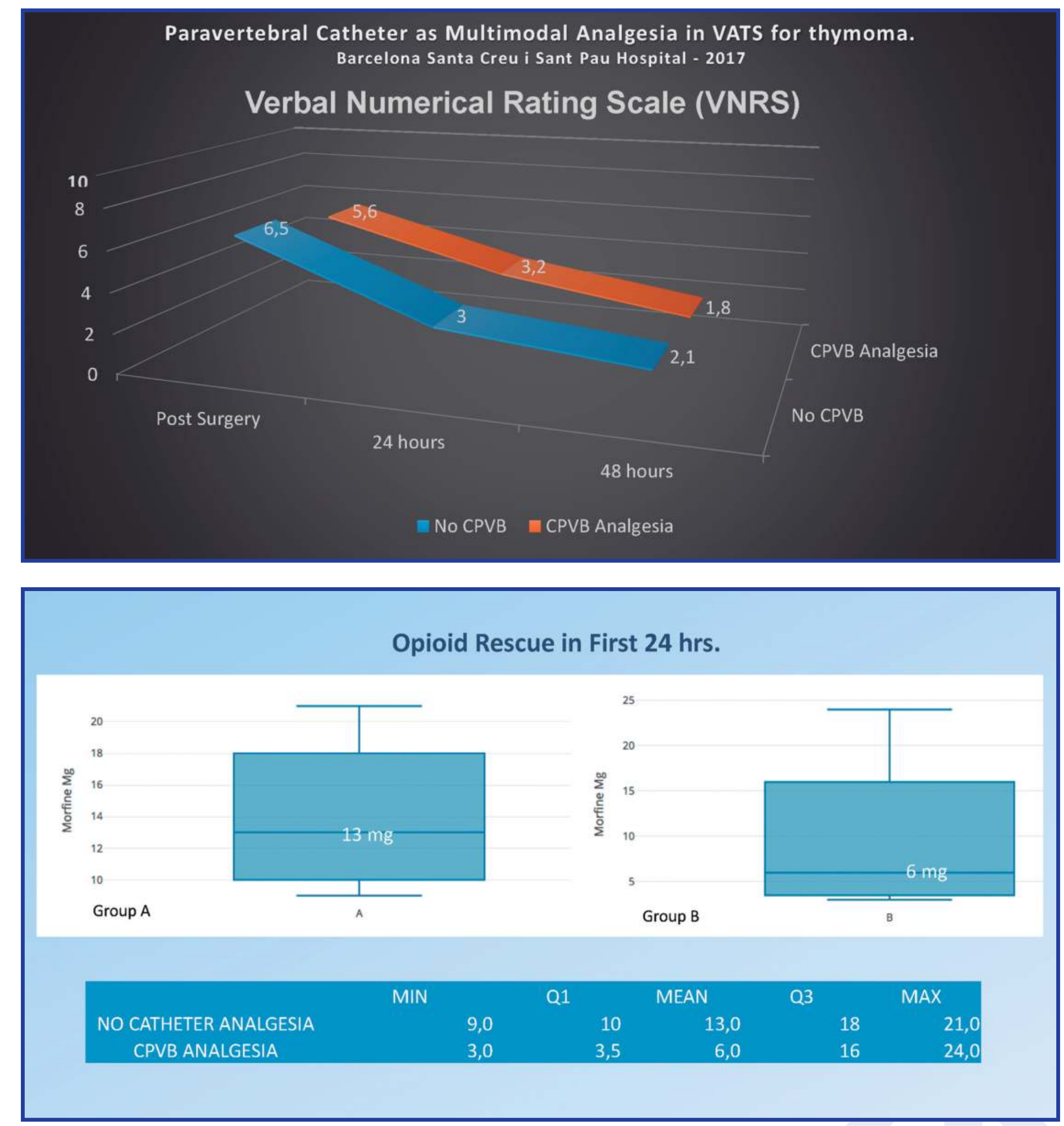

\section{Conclusion}

Multimodal analgesia approach with CPBV is a better alternative for myasthenia gravis in patients who undergo VATS thymectomy, decreasing the consumption of opioids in the first $\mathbf{2 4}$ hours postoperative and having a good pain relief. To avoid opioids is an important issue considering the neurologic characteristics of this patients. However, we need to perform prospective studies that confirm these preliminary results.

1. Sonett, JR, Magee MJ, et. Al. Thymectomy and myasthenia gravis: A history of surgical passion and scientific excellence The Journal of Thoracic and Cardiovascular Surgery C July 2017: 306-309.

2. Wolfe GI, Kaminski HJ, Aban IB, Minisman G, H.-C. Kuo et al. Randomized Trial of Thymectomy in Myasthenia Gravis N Engl J Med N Engl J Med 2016;375:511-22. DOI: 10.1056/NEJMoa1602489.

3. Yamauchi et al. Continuous paravertebral block using a thoracoscopic catheter-insertion technique for postoperative pain after thoracotomy: a retrospective case-control study Journal of Cardiothoracic Surgery (2017) 12:5. DOI 10.1186/s13019-017-0566-8.

4. Parera et. Al. Ultrasound-guided paravertebral catheter for postoperative analgesia in Video-Assisted thoracoscopic surgery. Euroanesthesia 2018. 